\title{
Research on Defect Diagnosis Method of Reactor Acoustic Vibration Method Based on Deep Learning
}

\author{
Peng-fei JIA ${ }^{1, *}$, Shu-guo $\mathrm{GAO}^{2}$, Xing-hui ZHANG ${ }^{3}$, Ling-ming $\mathrm{MENG}^{2}$, Yang YANG ${ }^{1}$ and Li-hua $\mathrm{LI}^{1}$ \\ ${ }^{1}$ China Electric Power Research Institute, High Voltage Research Institute, 15 Xiaoying East Road, Qinghe, Haidian District, Beijing, \\ China \\ ${ }^{2}$ Electric Power Research Institute, Hebei Electric Power Co., Ltd, 238 South TIYU street, Shijiazhuang, China \\ ${ }^{3}$ Equipment Management Department, State Grid Corporation Limited, 86 West Chang'an Street, Xicheng District, Beijing, China
}

\begin{abstract}
Although the state evaluation method based on characteristic parameters and weight factors can extract the characteristic quantities in time domain and frequency domain according to the collected acoustic and vibration signals of reactors, it is necessary to analyze a large number of test data to establish the functional relationship between the characteristic quantities and the defect states, and to establish the function relationship between the characteristic quantities and the defect states, and to establish the function relationship between the characteristic quantities and the defect states The method can directly learn the data samples, and self-study the correlation rules of characteristic parameters and defects through the training of neural network. In this paper, the deep learning neural network model is constructed, and the data obtained from reactor defect simulation experiment and field measurement are used as samples to train the deep learning network. Through the training of neural network, the characteristics of acoustic vibration signal are automatically learned, and the characteristics are stored in the parameters of neural network. Finally, the state of reactor is realized by the classifier at the end of the network assessment.
\end{abstract}

\section{Introduction}

For acoustic signal and vibration signal, the common fault diagnosis methods are based on analytical model, signal processing and artificial intelligence. The method based on analytical model is one of the earliest developed and most systematic fault diagnosis methods ${ }^{[1]}$. This method needs to diagnose and process the measured information according to certain mathematical methods on the basis of the mathematical model of the diagnosis object ${ }^{[2]}$. Its advantage is that it can go deep into the dynamic nature of the system essence and real-time diagnosis, and has inherent sensitivity to unknown faults, but its disadvantage is usually difficult Because of modeling error, disturbance and noise, the problem of robustness becomes more and more prominent. For the reactor, it is obviously unrealistic to establish its analytical model, so this method can not be applied ${ }^{[3]}$.

The fault diagnosis method based on signal analysis is to use the signal analysis theory to obtain a variety of deep-seated eigenvectors in time domain and frequency domain, and use the relationship between these eigenvectors and system fault sources to determine the location of fault sources ${ }^{[4]}$. This method is mainly used in the system where the analytical model of the diagnosis object is difficult to establish, but some state or output parameters of the system can be measured. The advantage of this method is that the signal model can be directly used without the mathematical model of the object, thus avoiding the difficulty of establishing the mathematical model of the object. The disadvantage is that the prior knowledge of the characteristic vector and fault relationship is needed.

With the rapid development of artificial intelligence and computer technology, a new theoretical basis for fault diagnosis technology has been provided. There are fault diagnosis methods based on knowledge and without precise mathematical model of the object ${ }^{[5]}$. There are diagnosis methods based on neural network, diagnosis method based on rough set, genetic algorithm, diagnosis method based on fault tree, etc. these methods do not need to extract features However, a large number of fault data are needed to train the diagnosis model.

In essence, deep learning is a solution. This scheme can make the computer learn from the existing experience, and the concept system can be divided into layers, so as to deepen the computer's understanding of the material world. Each concept is defined by its connection with some relatively simple concepts. Deep learning enables the computer to acquire knowledge from experience, which can avoid the formal specification of all the knowledge required by the computer.

Deep learning is a kind of machine learning, which has strong ability and flexibility. It formed several key concepts in the period of connectionism in the 1980s. The distributed representation can use linear parameters to represent regions of exponential numbers. With distributed representation, you can learn very complex

\footnotetext{
${ }^{*}$ Corresponding author:jiapengfei@epri.sgcc.com.cn
} 
functions with fewer instances. With the increasing amount of data, the significant increasing trend of data is driven by the increasingly digital society. Due to the increasing scale of the Internet, records are more easily organized into data sets for machine learning applications. With the increasing scale of models, the existing computing resources can run larger models. From the connectionist point of view, when many neurons in an animal work together, they become smarter and more effective than neurons alone or chickens and chickens. With the increasing precision, complexity and impact on the real world, the ability of deep learning to provide accurate identification and prediction has been improving since the 1980s. And more and more widely used in the depth of practical learning.

Due to its high flexibility, high autonomy, high accuracy and high learning, the defect early warning technology based on deep learning has more advantages than other defect early warning technologies.

For the acoustic vibration detection system operating in the actual working condition, the biggest difficulty is that there is no normal operation reactor acoustic vibration data and reactor acoustic vibration data under different operating conditions, especially the reactor acoustic vibration data under defect state is relatively small. In this case, the deep learning method is more suitable because it does not need a large number of training samples Use.

\section{Diagnosis of typical mechanical defects of reactor}

Although the state evaluation method based on characteristic parameters and weight factors can extract the characteristic quantities in time domain and frequency domain according to the collected acoustic and vibration signals of reactors, it is necessary to analyze a large number of test data to establish the functional relationship between the characteristic quantities and the defect states, and to establish the function relationship between the characteristic quantities and the defect states, and to establish the function relationship between the characteristic quantities and the defect states The method can directly learn the data samples, and self-study the correlation rules of characteristic parameters and defects through the training of neural network.

In this paper, the deep learning neural network model is constructed, and the data obtained from reactor defect simulation experiment and field measurement are used as samples to train the deep learning network. Through the training of neural network, the characteristics of acoustic vibration signal are automatically learned, and the characteristics are stored in the parameters of neural network. Finally, the state of reactor is realized by the classifier at the end of the network assessment.
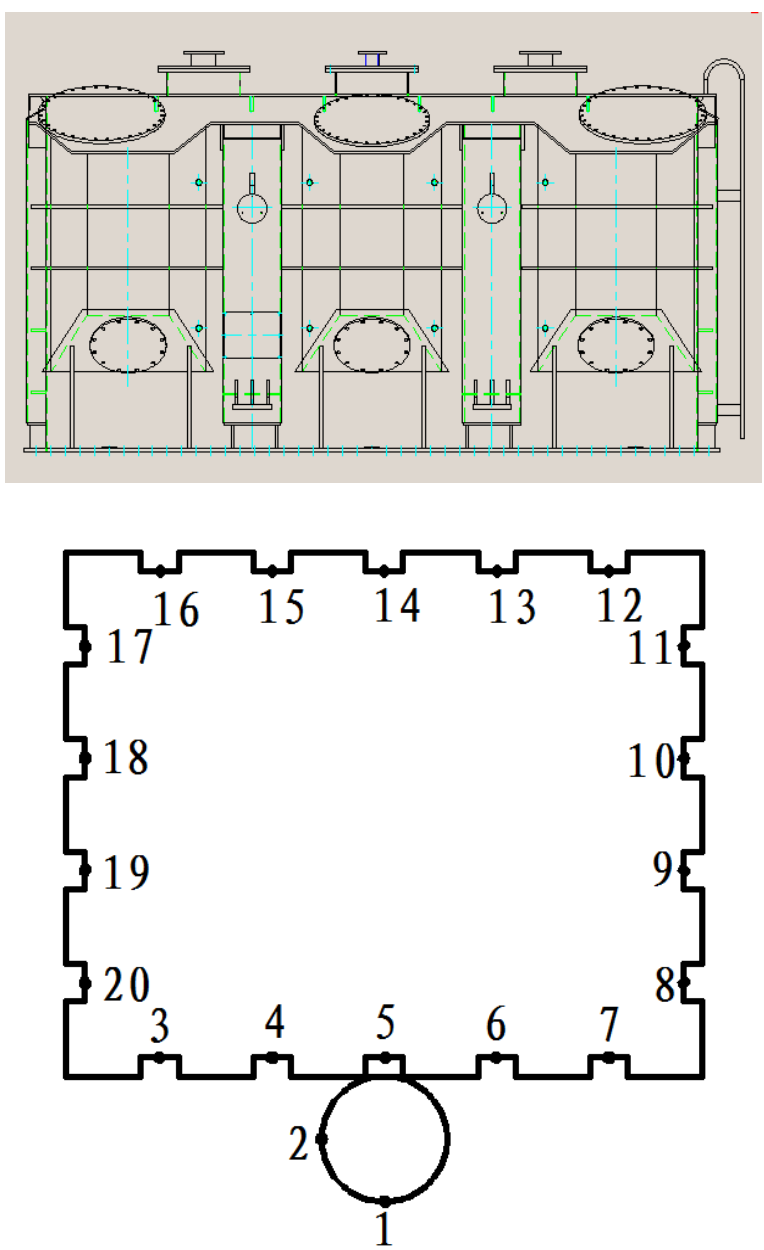

Fig. 1. Reactor outline and measuring point

\section{Reactor condition evaluation method based on deep learning}

$\mathrm{T}$ Referring to the successful application experience of deep learning in image processing, this project regards the combination of all acoustic vibration measurement points as a frame image, and each acoustic measurement point and vibration measurement point is regarded as a pixel block, and the method of convolution neural network is used for deep learning of acoustic and vibration signals of reactor. The input of neural network model is the data collected from acoustic signal and vibration signal measurement points. The input data is extracted features through convolution layer, and then the "activated neuron features" are preserved and mapped by activation function. Then, the feature dimension of convolution layer output is reduced by pooling layer, and the over fitting phenomenon of neural network is suppressed. Finally, the full connection layer of the network acts as a classifier in the whole convolution neural network, mapping the vibration signal of the acoustic signal of the reactor to the state of the reactor. The multi-dimensional acoustic and vibration signals are mapped to one-dimensional reactor state vector through the whole convolution neural network. 


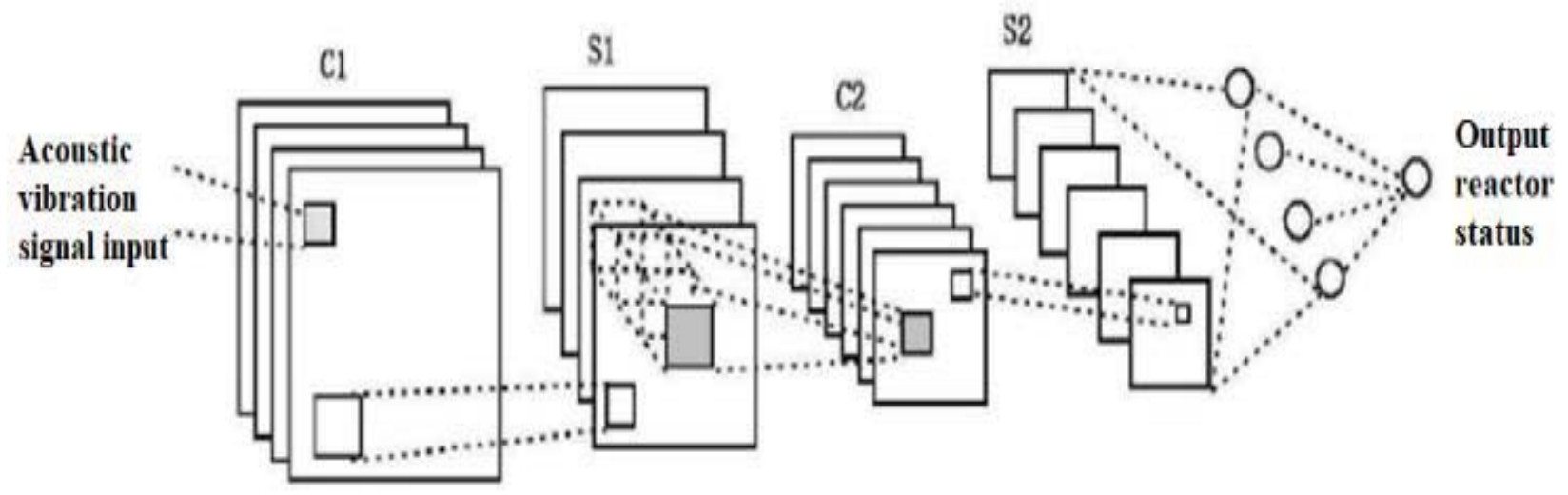

\section{\begin{tabular}{l|l|l|l|l|l|l|l|l|l|l} 
Convolution layer & Pool layer & Convolution layer & Pool layer & Full connection layer
\end{tabular}}

Fig. 2. Structure diagram of reactor acoustic vibration signal deep learning network

The structure of convolution neural network is similar to that of traditional neural network. The network consists of input layer, multi-layer hidden layer and output layer. There is no connection between neurons in each layer, and there is full connection between layers. The network model is shown in the figure. Convolution neural network model is essentially a kind of mapping relationship, which can be obtained by learning a large number of samples. During training, label samples are used for supervised learning. The basic algorithm includes two aspects: forward propagation of signal and backward propagation of error. In other words, the actual output is calculated according to the direction from input to output, while the correction of weight and threshold is carried out from the direction of output to input.

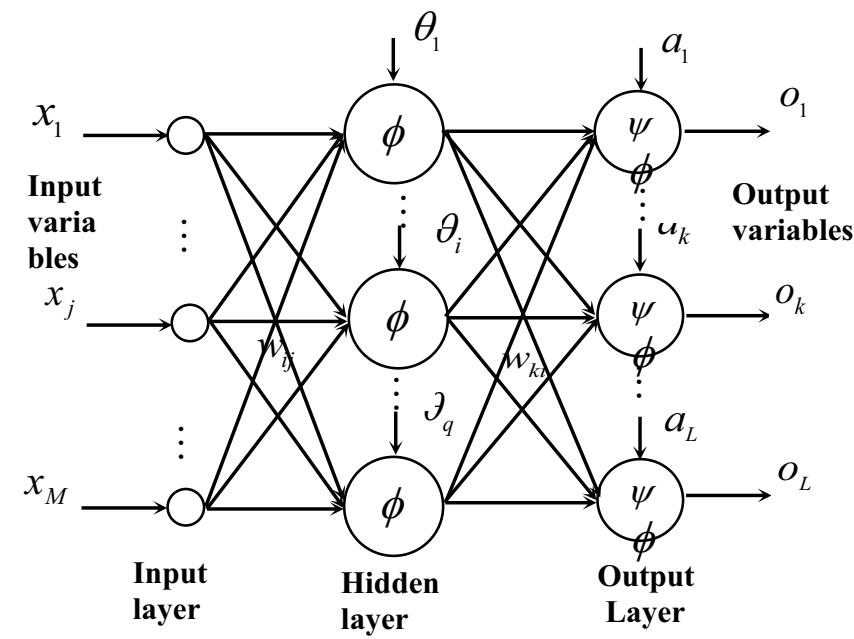

Fig. 3. Deep learning network structure

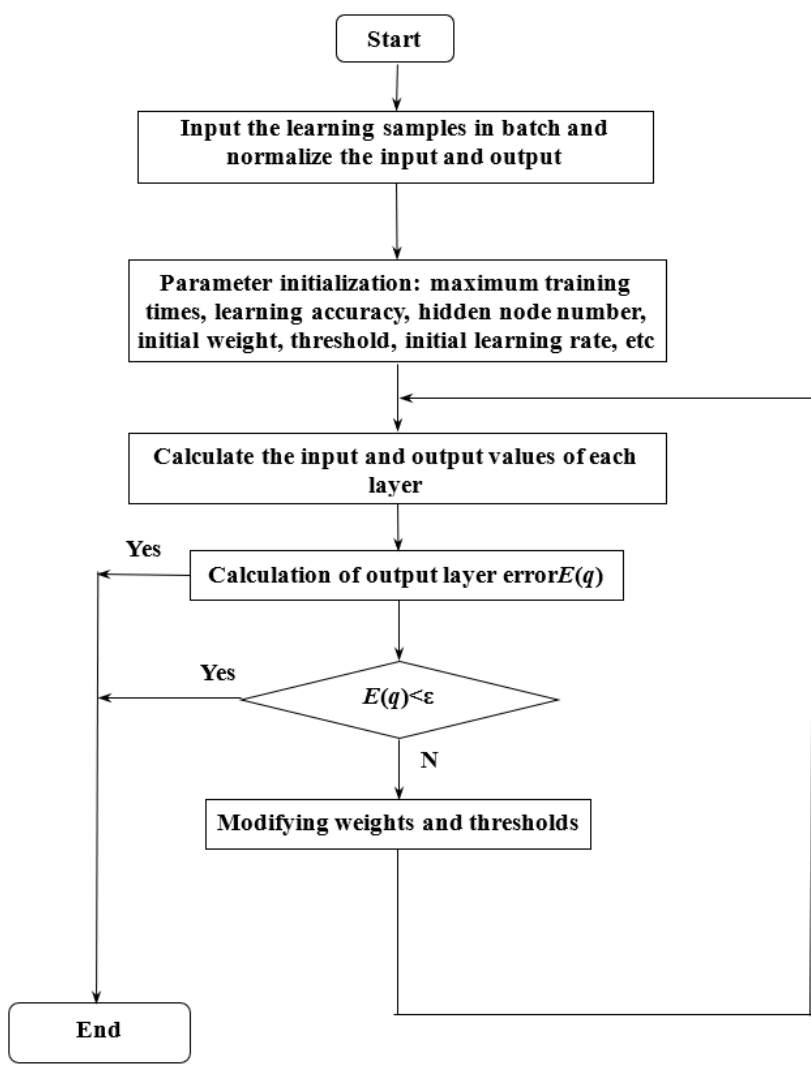

Figure 4. Neural network training algorithm program flow chart

$$
n t_{i}=\sum_{j=1}^{M} w_{i j} x_{j}+\theta_{i}
$$

$$
\begin{gathered}
y_{i}=\phi\left(\text { net }_{i}\right)=\phi\left(\sum_{j=1}^{M} w_{i j} x_{j}+\theta_{i}\right) \\
n e t_{k}=\sum_{i=1}^{q} w_{k i} y_{i}+a_{k}=\sum_{i=1}^{q} w_{k i} \phi\left(\sum_{j=1}^{M} w_{i j} x_{j}+\theta_{i}\right)+a_{k} \\
o_{k}=\psi\left(\text { net }_{k}\right)=\psi\left(\sum_{i=1}^{q} w_{k j} y_{i}+a_{k}\right)=\psi\left(\sum_{i=1}^{q} w_{k} \phi\left(\sum_{j=1}^{M} w_{i} x_{j}+\theta_{i}\right)+a_{k}\right)
\end{gathered}
$$




$$
\begin{aligned}
& E_{p}=\frac{1}{2} \sum_{k=1}^{L}\left(T_{k}-o_{k}\right)^{2} \\
& E=\frac{1}{2} \sum_{p=1}^{p} \sum_{k=1}^{L}\left(T_{k}^{p}-o_{k}^{p}\right)^{2} \\
& \Delta w_{k i}=-\eta \frac{\partial E}{\partial w_{k i}} ; \quad \Delta a_{k}=-\eta \frac{\partial E}{\partial a_{k}} ; \quad \Delta w_{i j}=-\eta \frac{\partial E}{\partial w_{i j}} ; \quad \Delta \theta_{i}=-\eta \frac{\partial E}{\partial \theta_{i}} \\
& \Delta w_{k i}=-\eta \frac{\partial E}{\partial w_{k i}}=-\eta \frac{\partial E}{\partial n e t_{k}} \frac{\partial n e t_{k}}{\partial w_{k i}}=-\eta \frac{\partial E}{\partial o_{k}} \frac{\partial o_{k}}{\partial n e t_{k}} \frac{\partial n e t_{k}}{\partial w_{k i}} \\
& \Delta a_{k}=-\eta \frac{\partial E}{\partial a_{k}}=-\eta \frac{\partial E}{\partial n e t_{k}} \frac{\partial n e t_{k}}{\partial a_{k}}=-\eta \frac{\partial E}{\partial o_{k}} \frac{\partial o_{k}}{\partial n e t_{k}} \frac{\partial n t_{k}}{\partial a_{k}} \\
& \Delta w_{i j}=-\eta \frac{\partial E}{\partial w_{i j}}=-\eta \frac{\partial E}{\partial n e t_{i}} \frac{\partial n e t_{i}}{\partial w_{i j}}=-\eta \frac{\partial E}{\partial y_{i}} \frac{\partial y_{i}}{\partial n e t_{i}} \frac{\partial n e t_{i}}{\partial w_{i j}} \\
& \Delta \theta_{i}=-\eta \frac{\partial E}{\partial \theta_{i}}=-\eta \frac{\partial E}{\partial \text { net }_{i}} \frac{\partial \text { net }_{i}}{\partial \theta_{i}}=-\eta \frac{\partial E}{\partial y_{i}} \frac{\partial y_{i}}{\partial \text { net }_{i}} \frac{\partial \text { net }_{i}}{\partial \theta_{i}} \\
& \frac{\partial E}{\partial o_{k}}=-\sum_{p=1}^{P} \sum_{k=1}^{L}\left(T_{k}^{p}-o_{k}^{p}\right) \\
& \frac{\partial n t_{k}}{\partial w_{k i}}=y_{i}, \quad \frac{\partial n e t_{k}}{\partial a_{k}}=1, \quad \frac{\partial n t_{i}}{\partial w_{i j}}=x_{j}, \quad \frac{\partial n e t_{i}}{\partial \theta_{i}}=1 \\
& \frac{\partial E}{\partial y_{i}}=-\sum_{p=1}^{P} \sum_{k=1}^{L}\left(T_{k}^{p}-o_{k}^{p}\right) \cdot \psi^{\prime}\left(\text { net }_{k}\right) \cdot w_{k i} \\
& \frac{\partial y_{i}}{\partial \text { net }_{i}}=\phi^{\prime}\left(\text { net }_{i}\right) \\
& \frac{\partial o_{k}}{\partial n e t_{k}}=\psi^{\prime}\left(\text { net }_{k}\right) \\
& \Delta w_{k i}=\eta \sum_{p=1}^{P} \sum_{k=1}^{L}\left(T_{k}^{p}-o_{k}^{p}\right) \cdot \psi^{\prime}\left(\text { net }_{k}\right) \cdot y_{i} \\
& \Delta a_{k}=\eta \sum_{p=1}^{P} \sum_{k=1}^{L}\left(T_{k}^{p}-o_{k}^{p}\right) \cdot \psi^{\prime}\left(n e_{k}\right) \\
& \Delta w_{i j}=\eta \sum_{p=1}^{p} \sum_{k=1}^{L}\left(T_{k}^{p}-o_{k}^{p}\right) \cdot \psi^{\prime}\left(n e t_{k}\right) \cdot w_{k i} \cdot \phi^{\prime}\left(n e t_{i}\right) \cdot x_{j} \\
& \Delta \theta_{i}=\eta \sum_{p=1}^{P} \sum_{k=1}^{L}\left(T_{k}^{p}-o_{k}^{p}\right) \cdot \psi^{\prime}\left(\text { net }_{k}\right) \cdot w_{k i} \cdot \phi^{\prime}\left(\text { net }_{i}\right)
\end{aligned}
$$

\section{Conclusion}

In this paper, the deep learning neural network model is constructed, and the data obtained from reactor defect simulation experiment and field measurement are used as samples to train the deep learning network. Through the training of neural network, the characteristics of acoustic vibration signal are automatically learned, and the characteristics are stored in the parameters of neural network. Finally, the state of reactor is realized by the classifier at the end of the network assessment.

We thank the State Grid Corporation of Science and Technology Project Research on Defect Detection Technology and Diagnosis Method of High Voltage Shunt Reactor Based on Acoustic Vibration Signal (grant number 5200201913054A-0-0-00).

\section{References}

1. YANHUI GAO, KAZUHIRO MURAMATSU, KOJI FUJIWARA, et al. Vibration Analysis of a Reactor Driven by an Inverter Power Supply Considering Electromagnetism and Magnerostriction[J]. IEEE TRANSACTIONS ON MAGNETICS, 2009, 45: 4789-4792.

2. WATARU KITAGAWA, YOSHIYUKI ISHIHARA, TOSHIYUKI TODAKA, et al. Analysis of Structural Deformation and Vibration of a Transformer Core by Using Magnetic Property of Magnetostriction[J]. Electrical Engineering in Japan, 2010, 172: 19-26.

3. Krizhevsky A, Sutskever I, Hinton G E. ImageNet classification with deep convolutional neural networks[C]. International Conference on Neural Information Processing Systems Curran Associates Inc. 2012: 1097-1105.

4. Zeiler M D, Fergus R. Visualizing and Understanding Convolutional Networks[J]. Lecture Notes in Computer Science, 2013, 8689: 818-833.

5. He K, Zhang X, Ren S, et al. Deep Residual Learning for Image Recognition[C]. Computer Vision and Pattern Recognition. IEEE, 2016: 770778 . 